OPEN ACCESS

Edited by:

Qing Zhao,

Second Affiliated Hospital

of Guangzhou Medical University,

China

Reviewed by:

$\mathrm{LiHu}$,

Chinese Academy of Sciences (CAS),

China

Kevin Fitzpatrick,

University of Arkansas, United States

${ }^{*}$ Correspondence: Kunming Tian

non-standstill@163.com

Yong Gan

scswj2008@163.com

Specialty section:

This article was submitted to

Health Psychology,

a section of the journal

Frontiers in Psychology

Received: 13 October 2020 Accepted: 17 November 2020

Published: 23 December 2020

Citation:

Peng S, Lai X, Du Y, Li Y, Tian K and Gan $Y$ (2020) Prevalence and Associated Factors

for Depressive Symptomatology in Chinese Adults During COVID-19 Epidemic. Front. Psychol. 11:616723.

doi: 10.3389/fpsyg.2020.616723

\section{Prevalence and Associated Factors for Depressive Symptomatology in Chinese Adults During COVID-19 Epidemic}

\author{
Songxu Peng ${ }^{1}$, Xin Lai ${ }^{1}$, Yukai Du' ${ }^{2}$, Yuting Li ${ }^{3}$, Kunming Tian ${ }^{4,5 *}$ and Yong Gan ${ }^{6 *}$
}

1 Department of Maternal and Child Health, Xiangya School of Public Health, Central South University, Changsha, China, ${ }^{2}$ Department of Maternal and Child Health, School of Public Health, Tongji Medical College, Huazhong University of Science and Technology, Wuhan, China, ${ }^{3}$ Cancer Center, Union Hospital, Tongji Medical College, Huazhong University of Science and Technology, Wuhan, China, ${ }^{4}$ Department of Preventive Medicine, School of Public Health, Zunyi Medical University, Zunyi, China, ${ }^{5}$ Institute of Reproductive Health, Tongji Medical College, Huazhong University of Science and Technology, Wuhan, China, ${ }^{6}$ Department of Social Medicine and Health Management, School of Public Health, Tongji Medical College, Huazhong University of Science and Technology, Wuhan, China

Background: The coronavirus disease 2019 (COVID-19) has been rapidly transmitted worldwide, which contributed to various psychological problems (such as fear, depression, and anxiety) among the general population in China. The purpose of this study is to investigate the prevalence and associated factors of depressive symptoms among Chinese adults.

Methods: A cross-sectional study of Chinese adults was conducted during 1729 February 2020. Symptoms of depression were assessed using the Center for Epidemiologic Studies Depression scale (CES-D).

Results: A total of 3,399 respondents were included in the analysis. It was observed that $14.2 \%(481 / 3,399)$ of the participants were screened positive for depressive symptoms. In a multivariate logistic regression analysis, older age $(\mathrm{OR}=0.98 ; 95 \%$ $\mathrm{Cl}$, 0.97-0.99), smoking ( $\mathrm{OR}=1.57 ; 95 \% \mathrm{Cl}, 1.10-2.26)$, self-rated health (good: $\mathrm{OR}=0.49 ; 95 \% \mathrm{Cl}, 0.37-0.66$; fairly: $\mathrm{OR}=0.60 ; 95 \% \mathrm{Cl}, 0.45-0.80)$, having greater support scores $(\mathrm{OR}=0.95 ; 95 \% \mathrm{Cl}, 0.94-0.96)$, knowledge about the main symptom of COVID-19 (very clearly: $\mathrm{OR}=0.58 ; 95 \% \mathrm{Cl}, 0.42-0.79$; relatively clearly: $\mathrm{OR}=0.59$; 95\% Cl, 0.44-0.79), and staying in Wuhan within 3 months before the outbreak of epidemic (OR $=1.78 ; 95 \% \mathrm{Cl}, 1.34-2.38)$ were associated with depressive symptoms.

Conclusion: A considerable proportion of the general population in China had depressive symptoms during the COVID-19 epidemic. Routine screening and targeted interventions for depression are needed among high-risk depressed individuals during the COVID-19 epidemic.

Keywords: epidemic, COVID-19, risk factors, prevalence, depression symptoms 


\section{BACKGROUND}

The coronavirus disease 2019 (COVID-19) spread rapidly worldwide, causing high morbidity and heavy economic burden (Docherty et al., 2020; Karagiannidis et al., 2020; Onder et al., 2020; Tadesse et al., 2020; Tang et al., 2020; Wu and McGoogan, 2020; Loomba et al., 2021). In December 2019, a pneumonia of unknown cause was detected and became an epidemic in Wuhan, China. On 7 January 2020, the severe acute respiratory syndrome coronavirus 2 (SARS-CoV2) was identified as the etiological agent of the epidemic by genome sequencing ( $\mathrm{Zhu}$ et al., 2020). Because of the quick spreading of the virus in multiple countries, at the meeting of the emergency committee of the World Health Organization (WHO) on 30 January 2020, COVID-19 was declared a Public Health Emergency of International Concern (PHEIC) (World Health Organization, 2020a). Subsequently, WHO formally named COVID-19 on 11 February 2020 (World Health Organization, 2020b). According to the official website of the National Health Commission, on 17 February 2020, a total of 72,436 confirmed cases and 1,868 death cases were identified in China, with a fatality rate of $2.6 \%$ (The National Health Commission of China, 2020). The COVID-19 outbreak posed great threat to human life (Wang et al., 2020a). Additionally, findings from previous studies suggested that outbreaks of major communicable diseases, including severe acute respiratory syndrome and Middle East respiratory syndrome, increased the risk of depression in the populations affected (Mak et al., 2009; Keita et al., 2017). Compared with previous epidemics and pandemics, COVID-19 is more contagious and spreads faster (Meo et al., 2020), which may further aggravate the public's depression. Therefore, timely psychological assessment and appropriate intervention to prevent depressive symptom is necessary.

Depression is a common mood disorder characterized by persistently low mood state, affecting $1.4-37.5 \%$ of the population (Maske et al., 2016; Rotenstein et al., 2016; Arias-de la Torre et al., 2018). Several studies had shown that depression not only reduces the quality of life of patients but also increases the risk of chronic physical diseases and suicide (Whooley and Wong, 2013; Kozela et al., 2016; Lopez et al., 2018). Recent studies have shown that the prevalence of depressive symptoms in the general population is high during the COVID19 epidemic, ranging from 3.7 to 68\% (Ettman et al., 2020; Goularte et al., 2020; Rodríguez-Rey et al., 2020; Tan et al., 2020; van der Velden et al., 2020). A published meta-analysis study included 14 studies and suggested that $33.7 \%$ of the general population had depressive symptoms during the COVID19 pandemic (Salari et al., 2020). Additionally, several Chinese studies reported the prevalence of depressive symptoms in the general population during the COVID-19 outbreak. For example, a survey study conducted by Shi et al. (2020) with 56,679 Chinese participants reported $27.9 \%$ of the general population had depressive symptoms. A previous study from Shenzhen suggested that the prevalence of depressive symptom was $6.2 \%$ in the general population quarantined (Peng et al., 2020), performing in subjects with quarantine experience.
Another study by Lei et al. (2020) found the prevalence of depression was approximately $14.6 \%$, with mixed samples including people affected and people unaffected. Therefore, the prevalence of depressive symptoms among the general population is still inconsistent, probably due to the different population and sampling issues in these studies, which deserves further study.

In the past decades, risk factors for depressive symptoms have frequently been examined (Hammen, 2018). However, few studies have explored epidemic-related factors causing depressive symptoms. As a newly emerging disease, the public has limited knowledge about main symptoms, latent period, and route of transmission of COVID-19, and the number of confirmed cases varies from region to region, which may influence the degree of depressive symptoms in the general population. Additionally, Wuhan, as the center of the epidemic in China, whether people with a history of exposure to Wuhan before the COVID-19 epidemic outbreak have a higher risk of depression, it is unclear. To better allocate limited medical resources and formulate effective interventions, the prevalence of depressive symptoms and epidemic-related factors that are associated with them during the COVID-19 outbreak need to be determined urgently.

Therefore, we enrolled a large number of participants and investigated sociodemographic information and epidemicrelated factors, which would provide a more complete overview of the risk factors of depressive symptoms. This study aimed to investigate the prevalence and identify the associated factors of depressive symptoms among the general public during COVID19 epidemic.

\section{MATERIALS AND METHODS}

\section{Study Design and Participants}

We conducted a cross-sectional online survey to assess the prevalence and risk factors of depressive symptoms among the general population in China during the COVID-19 epidemic. An online survey was used because of the self-isolation of the general population in China. We adopted snowball sampling strategy to recruit participators; potential participators were electronically invited via WeChat. The recruitment information of the study was posted on the university website. Data collection was completed during 17-29 February 2020 period. This survey period corresponded to the reduction stage after the peak point of the COVID-19 epidemic outbreak in the country.

The present study was approved by the Institutional Review Board of Tongji Medical College, Huazhong University of Science and Technology. Informed consent was obtained from the participants before their participation in the study.

\section{Data Collection}

A self-administered questionnaire was used to collect data on sociodemographic characteristics, knowledge about COVID19, information regarding depressive symptoms and social support, and additional information related to COVID-19. Sociodemographic data included age (continuous variable, ranged from 18 to 99), gender (dummy coded, $0=$ female, 
$1=$ male), nationality (dummy coded, $0=$ Han, $1=$ Other), marital status (dummy coded, $0=$ unmarried, $1=$ married), employment status (dummy coded, $0=$ unemployed, 1 = employed), education (dummy coded, $0=$ high school and below, 1 = college, 2 = bachelor or above), type of residence (dummy coded, $0=$ urban, $1=$ rural, $2=$ urban-rural district), smoking (dummy coded, $0=$ non-smoker, $1=$ smoker) and drinking status (dummy coded, $0=$ non-drinking, $1=$ drinking), and history of chronic diseases (dummy coded, $0=$ no, $1=$ yes). Respondents were asked to rate their physical health status (dummy coded, 0 = average/poor, 1 = fairly good, 2 = very good). Knowledge about COVID-19 included the main symptom, latent period, and routes of transmission of COVID-19 (dummy coded, 0 = general, 1 = relatively clear, 2 = very clear). Additionally, respondents were asked to answer if they stayed in Wuhan within 3 months before the epidemic outbreak or had any relatives or friends who were infected with COVID-19 (dummy coded, 0 = no, 1 = yes). Depressive symptoms and social support were assessed by the Center for Epidemiologic Studies Depression scale (CES-D) and Perceived Social Support Scale (PSSS), respectively.

\section{Social Support}

The PSSS was designed by Zimet et al. (1990). The scale has 12 items including three dimensions, namely, family support, friend support, and other support. It is scored on a 7-point Likerttype scale, with higher scores indicating higher perception of social support. The Chinese version of PSSS has been proven to be highly reliable and valid among the Chinese population (Ye et al., 2017). Here, the Cronbach's alpha value was 0.96.

\section{Depression Symptoms}

The CES-D was used to assess depressive symptoms. It comprises 20 items; each item is rated on a 4-point scale ranging from 0 ("rarely or none of the time") to 3 ("most or almost all of the time"). The total score ranges from 0 to 60 , with a higher score indicating more severe depressive symptoms. For the original CES-D scale, a total score of 16 is used to detect the presence of depressive symptoms (Radloff, 1977). However, a large number of studies have assessed the diagnostic accuracy of the CES-D to detect depression in the general population and proposed a variety of cut-off scores, such as a cut-off score of 18 among elders living in residential homes (Dozeman et al., 2011) and a cut-off score of 22 in older Chinese (Cheng and Chan, 2005). A meta-analytic study systematically reviewed 28 CES-D studies including several Chinese studies and proposed an optimal cut-off score of 20 (Vilagut et al., 2016). Hence, a cutoff value of 20 or a greater total score was considered indicative of depressive symptoms, consistent with the previous research (Jiang et al., 2019). This Chinese version of the CES-D has good reliability and validity and has been widely adopted in the Chinese population. In the present study, the Cronbach's alpha coefficient for this scale was 0.96 .

\section{Statistical Analysis}

Continuous variables were expressed as the mean \pm standard deviation (SD) and compared using Student's $t$-tests. Categorical data were presented as percentages and compared using Chisquare tests. A multivariable logistical regression model was used to identify the risk factors for depression. Statistical significance was assessed at 5\% level (two-tailed test). All analyses were performed using SPSS software version 18.0 (SPSS, Chicago, IL, United States).

\section{RESULTS}

\section{Characteristics of Study Population}

A total of 3,405 completed questionnaires were received, and six respondents who lived abroad were excluded from this study. Finally, 3,399 respondents were included in the analysis. The 3,399 respondents from 280 cities in China had an average age of 27.5 years (range, 18-80) with standard deviation of 11.4 years; $95.5 \%$ of them were of Han nationality, and $66.5 \%$ were female.

Based on the presence of depressive symptoms, all participants were categorized into two groups: the depression and nondepression groups; $14.2 \%(481 / 3,399)$ of the participants were screened positive for depressive symptoms in the present study and included in the depression group. Table 1 compares the sociodemographic and behavior characteristics of participants with and without depressive symptoms. Participants in the depression group were more likely to have a younger age, lower medical insurance coverage, worse self-rated health,

TABLE 1 | Demographic and behavior characteristics of participants with or without depressive symptoms.

\begin{tabular}{|c|c|c|c|c|}
\hline Characteristics & $\begin{array}{c}\text { Depression } \\
(n=481)\end{array}$ & $\begin{array}{l}\text { Non-depression } \\
(n=2,918)\end{array}$ & $t / x^{2}$ & $P$-value \\
\hline Age (years, mean $\pm S D$ ) & $26.3 \pm 12.5$ & $27.8 \pm 11.2$ & 2.42 & 0.016 \\
\hline Female (\%) & $299(62.1)$ & $1,962(67.2)$ & 4.78 & 0.029 \\
\hline Han nationality & 455 (94.6) & 2, $794(95.8)$ & 1.31 & 0.253 \\
\hline Married & $121(25.2)$ & $985(33.8)$ & 13.91 & 0.000 \\
\hline Educational level (years) & & & 0.47 & 0.790 \\
\hline High school and below & 55 (11.4) & 331 (11.3) & & \\
\hline College & $65(13.5)$ & $429(14.7)$ & & \\
\hline Bachelor or above & $361(75.1)$ & $2,158(74.0)$ & & \\
\hline Type of residence & & & 0.05 & 0.975 \\
\hline Urban & $282(58.6)$ & 1, $695(58.1)$ & & \\
\hline Rural & $154(32.0)$ & $946(32.4)$ & & \\
\hline Urban-rural district & $45(9.4)$ & $277(9.5)$ & & \\
\hline Employed & $163(33.9)$ & $1,167(40.0)$ & 6.46 & 0.011 \\
\hline Smoker (\%) & $51(10.6)$ & $226(7.7)$ & 4.51 & 0.034 \\
\hline Alcohol (\%) & $72(15.0)$ & 398 (13.6) & 0.61 & 0.434 \\
\hline Chronic disease & $49(10.2)$ & $240(8.2)$ & 2.04 & 0.153 \\
\hline $\begin{array}{l}\text { Medical insurance } \\
\text { coverage }\end{array}$ & $398(82.7)$ & 2, $544(87.2)$ & 6.99 & 0.008 \\
\hline Self-rated health & & & 57.09 & 0.000 \\
\hline Very good & 194 (40.3) & $1,527(52.3)$ & & \\
\hline Quite good & $170(35.3)$ & 1, $040(35.6)$ & & \\
\hline Average & 117 (24.3) & 351 (12.0) & & \\
\hline Support scores & $57.8 \pm 13.8$ & $67.4 \pm 11.6$ & 14.34 & 0.000 \\
\hline
\end{tabular}

SD, standard deviation. 
and lower support scores compared with those in the nondepression group. In contrast, participants in the non-depression group were likely to be married and employed, female, and non-smoker. However, there was no significant difference in nationality, education, types of residence, alcohol use, and history of chronic disease between the depression and nondepression groups.

\section{Comparison of Concerns and Knowledge About COVID-19 Among Depression and Non-depression Groups}

Regarding concerns and knowledge about COVID-19, Table 2 shows that participants in the depression group reported a higher exposure of staying in Wuhan within 3 months before the epidemic outbreak compared with those in the non-depression group. Additionally, participants in the depression group were less knowledgeable about the main symptoms, latent period, and routes of transmission of COVID-19 than those in the nondepression group.

\section{Multivariate Regression Analysis in Identifying Risk Factors of Depression}

This study used multivariate logistical regression analysis to identify risk factors of depressive symptoms. Variables with

TABLE 2 | Comparison of concerns and knowledge's of COVID-19 between people with or without depressive symptoms.

\begin{tabular}{|c|c|c|c|c|}
\hline Characteristics & $\begin{array}{l}\text { Depression } \\
(n=481)\end{array}$ & $\begin{array}{l}\text { Non-depression } \\
\quad(n=2,918)\end{array}$ & $x^{2}$ & $P$-value \\
\hline \multicolumn{3}{|l|}{ Local cases } & 2.14 & 0.544 \\
\hline$<500$ & $100(20.8)$ & 542 (18.6) & & \\
\hline $500-1,000$ & $271(56.3)$ & $1,743(59.7)$ & & \\
\hline $1,000-10,000$ & $53(11.0)$ & $304(10.4)$ & & \\
\hline$>10,000$ & $57(11.9)$ & $329(11.3)$ & & \\
\hline \multicolumn{3}{|c|}{$\begin{array}{l}\text { Staying in Wuhan during } 3 \text { months before the } \\
\text { outbreak of epidemic? }\end{array}$} & 6.74 & 0.009 \\
\hline Yes & $76(15.8)$ & $339(11.6)$ & & \\
\hline No & $405(84.2)$ & $2,579(88.4)$ & & \\
\hline \multicolumn{3}{|c|}{ Whether you or your relatives are confirmed by COVID-19? } & 2.13 & 0.144 \\
\hline Yes & $5(1.0)$ & $12(0.4)$ & & \\
\hline No & 476 (99.0) & 2, $906(99.6)$ & & \\
\hline \multicolumn{3}{|c|}{ Do you know the main symptom of COVID-19? } & 57.72 & 0.000 \\
\hline Very clear & $134(27.9)$ & 1, 098 (37.6) & & \\
\hline Relatively clear & 245 (50.9) & $1,533(52.5)$ & & \\
\hline General & $102(21.2)$ & $287(9.8)$ & & \\
\hline \multicolumn{3}{|c|}{ Do you know the latent period of COVID-19? } & 37.77 & 0.000 \\
\hline Very clear & $185(38.5)$ & 1, $446(49.6)$ & & \\
\hline Relatively clear & $229(47.6)$ & $1,270(43.5)$ & & \\
\hline General & 367 (13.9) & $202(6.9)$ & & \\
\hline \multicolumn{3}{|c|}{ Do you know the routes of transmission of COVID-19? } & 44.58 & 0.000 \\
\hline Very clear & $181(37.6)$ & 1, $396(47.8)$ & & \\
\hline Relatively clear & $234(48.6)$ & 1, $347(46.2)$ & & \\
\hline General & 66 (13.7) & 175 (6.0) & & \\
\hline
\end{tabular}

COVID-19, 2019 coronavirus disease. statistical significance in univariate analysis, including age, gender, marital status, employment status, smoking status, medical insurance coverage, self-rated health, support scores, staying in Wuhan within 3 months before the epidemic outbreak, and knowledge about the main symptoms, latent period, and routes of transmission of COVID-19, were included in the logistical regression model. Results showed that older age (OR $=0.98 ; 95 \% \mathrm{CI}, 0.97-0.99 ; P=0.000)$, very good self-rated health $(\mathrm{OR}=0.49 ; 95 \% \mathrm{CI}, 0.37-0.66 ; P=0.000)$, fairly good self-rated health $(\mathrm{OR}=0.60 ; 95 \% \mathrm{CI}, 0.45-0.80 ; P=0.001)$, greater support scores $(\mathrm{OR}=0.95 ; 95 \% \mathrm{CI}, 0.94-0.96 ; P=0.000)$, very clear knowledge about the main symptoms of COVID-19 $(\mathrm{OR}=0.58 ; 95 \% \mathrm{CI}, 0.42-0.79 ; P=0.001)$, and relatively clear knowledge about the main symptoms of COVID-19 (OR = 0.59; 95\% CI, 0.44-0.79; $P=0.000)$ were associated with a decreased risk of depressive symptoms. However, smoking $(\mathrm{OR}=1.57$; 95\% CI, 1.10-2.26; $P=0.014$ ) and staying in Wuhan within 3 months before the epidemic outbreak (OR $=1.78 ; 95 \% \mathrm{CI}$, 1.34-2.38; $P=0.000$ ) were associated with an increased risk of depressive symptoms (Table 3 ).

\section{DISCUSSION}

This cross-sectional study, based on 3,399 participants assessed the prevalence and risk factors of depressive symptoms among the Chinese general population during the COVID-19 epidemic. We found that $14.2 \%$ of the general population suffered from depressive symptoms during the COVID-19 epidemic. Furthermore, some demographic and concerns as well as knowledge about COVID-19 variables were found to be the influencing factors for depressive symptoms, including age, smoking status, self-rated health, social support, knowledge about the main symptoms of COVID-19, and staying in Wuhan within 3 months before the outbreak.

The prevalence of depressive symptoms among the general population in China during the COVID-19 epidemic in the present study is similar to that in recent published studies. The study by Wang et al. (2020b) with 1,210 respondents from 194 cities in China reported that $16.5 \%$ of the general population had moderate to severe depressive symptoms. Lei et al. (2020) reported that the prevalence of depressive symptoms in the public affected and those unaffected was $14.6 \%$ during the COVID-19 epidemic. However, a high prevalence of depressive symptoms among general population was observed in other countries, such as $16.9 \%$ in Netherlands (van der Velden et al., 2020), $27.8 \%$ in America (Ettman et al., 2020), 68\% in Brazil (Goularte et al., 2020), and 41\% in Spain (Rodríguez-Rey et al., 2020). This discrepancy in terms of prevalence of depression may be explained, to some extent, by differences in diagnostic criteria, severity of the epidemic, and intervention measures taken. The Chinese government had taken several measures to control and reduce COVID-19 transmission, such as city closure, traffic control, isolation at home, and wearing of masks. Majority of the Chinese general population considered that these measures were effective and believed that they would win the battle against COVID-19. 
TABLE 3 | Multivariable logistic analyses for factors related to depressive symptoms.

\begin{tabular}{|c|c|c|c|c|c|c|c|}
\hline & \multirow[t]{2}{*}{ Coefficients B } & \multirow[t]{2}{*}{ SE } & \multirow[t]{2}{*}{ Wald } & \multirow[t]{2}{*}{$P$-value } & \multirow[t]{2}{*}{ OR } & \multicolumn{2}{|c|}{$95 \%$ confidence interval for OR } \\
\hline & & & & & & Lower & Upper \\
\hline Age & -0.019 & 0.005 & 14.702 & 0.000 & 0.981 & 0.972 & 0.991 \\
\hline Smoking & 0.454 & 0.184 & 6.078 & 0.014 & 1.574 & 1.097 & 2.258 \\
\hline Self-rated health & & & 23.296 & 0.000 & & & \\
\hline Very good & -0.7 & 0.145 & 23.171 & 0.000 & 0.497 & 0.373 & 0.660 \\
\hline Fairly good & -0.511 & 0.147 & 12.039 & 0.001 & 0.600 & 0.450 & 0.801 \\
\hline Average/poor & & & & & Reference & & \\
\hline Support scores & -0.055 & 0.004 & 169.889 & 0.000 & 0.947 & 0.939 & 0.955 \\
\hline \multicolumn{3}{|c|}{ Do you know the main symptom of COVID-19? } & 14.583 & 0.001 & & & \\
\hline Very clear & -0.551 & 0.162 & 11.528 & 0.001 & 0.577 & 0.42 & 0.792 \\
\hline Relatively clear & -0.525 & 0.146 & 12.96 & 0.000 & 0.591 & 0.444 & 0.787 \\
\hline General & & & & & Reference & & \\
\hline Staying in Wuhan & 0.578 & 0.147 & 15.382 & 0.000 & 1.783 & 1.335 & 2.38 \\
\hline (Constant) & 2.98 & 0.309 & 93.239 & 0 & 19.698 & & \\
\hline
\end{tabular}

COVID-19, 2019 coronavirus disease; OR, odds ratio; Cl, confidence interval.

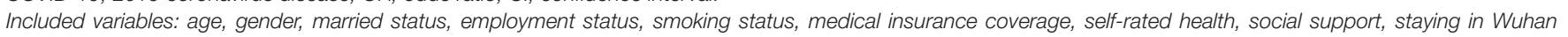
within 3 months before the outbreak of epidemic, knowledge's about the main symptoms, latent period, routes of transmission of COVID-19.

Our study found that younger individuals are more likely to have depressive symptoms compared with older individuals; similar results can be seen in the study of Huang and Zhao (2020), which concluded that younger people had a significantly higher prevalence of depressive symptoms than older people. However, most cross-sectional studies showed that higher age was associated with a higher risk of depressive symptoms in a non-disaster situation (Liu et al., 2015; Zhang et al., 2019). This discrepancy could be due to adaptive mechanisms, older people developed this ability to dealing with crises, which can be used to manage the stress associated with the current pandemic (Goularte et al., 2020). Additionally, young people tend to quickly obtain COVID-19 information from social media, which can easily trigger stress (Qiu et al., 2020).

The present study also showed that a higher prevalence of depressive symptoms was observed among individuals with a smoking habit, poor self-rated health, and low social support, which is consistent with most published studies (Pasco et al., 2008; Hu et al., 2018). Interestingly, depressive symptoms and the risk factors mentioned above had a bidirectional correlation. A 10 years study by Naicker et al. (2013) reported that depressed adolescents had higher proportion of smoking habit, poorer self-rated health, and lower social support compared with non-depressed adolescents 10 years later. Similarly, smoking habit, poor self-rated health, and low social support could increase the risk of depressive symptoms (Rousou et al., 2016; Holden et al., 2019).

Our study showed that individuals with an unclear understanding of the main symptoms of COVID-19 were more likely to report depressive symptoms, which is similar to the study of Wang et al. (2020b). The possible explanation for this phenomenon is that a more comprehensive understanding of the related knowledge about COVID-19 will help individuals face the epidemic rationally and judge effectively whether one is infected or not. Additionally, with a higher cognitive level regarding COVID-19, individuals would have more confidence and motivation to adopt effective measures to protect themselves. Therefore, improving public awareness of COVID-19 is necessary to improve mental health.

Our study suggested that staying in Wuhan within 3 months before the COVID-19 outbreak was associated with a higher risk of depressive symptoms. Lai et al. (2020) investigated 1,257 medical staff from fever clinics or wards of hospitals for patients with COVID-19 in China and reported that health-care workers in Wuhan had severe symptoms of depression, anxiety, insomnia, and distress. Wuhan is the origin and epicenter of the COVID-19 epidemic in China. Nearly $60 \%$ of the cases occurred in China, and a fatality rate of $5 \%$ was reported in Wuhan (Wu et al., 2020). Therefore, individuals with exposure in Wuhan before the COVID-19 outbreak were at an especially high risk for infection, which further increased the level of negative emotion, such as depression, anxiety, stress, and fear.

In this study, no significant correlation was observed between the number of local confirmed cases, COVID-19 infection status, COVID-19 latent period, route of transmission, and depressive symptoms. However, related studies have found that these factors may also affect the occurrence of depressive symptoms (Nelson et al., 2020; Zhang et al., 2020). This difference may be related to the study population and sampling methods. With the wide dissemination of information in China, the difference in the prevalence of depressive symptoms between severe and mild areas of the epidemic may have been weakened. Additionally, only 17 people reported that they or their relatives had COVID19 infection in this study, which may affect the reliability of the results. Therefore, it is necessary to explore this correlation in a large sample population.

This study had multiple strengths. Firstly, the study included a large number of subjects, which made it possible to obtain more convincing results. Secondly, this study explored the 
association between factors connecting COVID-19 epidemic to depressive symptoms.

However, there were limitations too. First, we adopted a snowball sampling strategy. The snowball sampling selects potential respondents based on existing respondents, which is not a random sampling method. The recruitment information of study was posted on the university website, resulting in most of the respondents coming from young students. Therefore, there was bias in the selection of participants in our study and the study's sample population may not be a good representation of the actual pattern of the general population. Second, our study was cross-sectional; we could not infer causal relationship of risk factors and symptoms of depression. Therefore, a cohort study is needed to certify this temporal relationship. Third, the CES$\mathrm{D}$ is just a screening tool and not a diagnostic one, although it has been widely used and validated in China. Fourth, this study adopted a self-administered online questionnaire, thus participants need to be able to use network tools, which might have affected how they responded to the questionnaire. However, given the COVID-19 epidemic, an online survey was considered more appropriate.

\section{CONCLUSION}

This study indicates that during the COVID-19 epidemic in China, a considerable proportion of the general population suffered from depressive symptoms. Those with younger age, a smoking habit, poorer self-rated health, and lower social support had a higher risk of depressive symptoms. Moreover, depressive symptoms were found to be significantly associated with knowledge about the main symptoms of COVID-19 and staying in Wuhan within 3 months before the COVID-19 outbreak. Given our findings, we recommend the establishment of targeted psychological interventions for the public to improve their mental health during the COVID-19 epidemic.

\section{REFERENCES}

Arias-de la Torre, J., Vilagut, G., Martin, V., Molina, A. J., and Alonso, J. (2018). Prevalence of major depressive disorder and association with personal and socio-economic factors. results for spain of the European Health Interview Survey 2014-2015. J. Affect. Disord. 239, 203-207. doi: 10.1016/j.jad.2018. 06.051

Cheng, S. T., and Chan, A. C. (2005). The center for epidemiologic studies depression scale in older Chinese: thresholds for long and short forms. Int. J. Geriatr. Psychiatry 20, 465-470. doi: 10.1002/gps.1314

Docherty, A. B., Harrison, E. M., Green, C. A., Hardwick, H. E., Pius, R., Norman, L., et al. (2020). Features of 20133 UK patients in hospital with covid-19 using the ISARIC WHO clinical characterisation protocol: prospective observational cohort study. BMJ 369:m1985. doi: 10.1136/bmj.m1985

Dozeman, E., van Schaik, D. J., van Marwijk, H. W., Stek, M. L., van der Horst, H. E., and Beekman, A. T. (2011). The center for epidemiological studies depression scale (CES-D) is an adequate screening instrument for depressive and anxiety disorders in a very old population living in residential homes. Int. J. Geriatr. Psychiatry 26, 239-246. doi: 10.1002/gps.2519

Ettman, C. K., Abdalla, S. M., Cohen, G. H., Sampson, L., Vivier, P. M., and Galea, S. (2020). Prevalence of depression symptoms in US Adults before and during the COVID-19 Pandemic. JAMA Netw. Open 3:e2019686. doi: 10.1001/ jamanetworkopen.2020.19686

\section{DATA AVAILABILITY STATEMENT}

The original contributions presented in the study are included in the article/supplementary material, further inquiries can be directed to the corresponding author/s.

\section{ETHICS STATEMENT}

The studies involving human participants were reviewed and approved by the Institutional Review Board of Tongji Medical College, Huazhong University of Science and Technology. The patients/participants provided their written informed consent to participate in this study.

\section{AUTHOR CONTRIBUTIONS}

SP participated all preparation of this manuscript. XL contributed to statistical analysis. YD and YL took part in the sample collection. KT and YG contributed to study design and the critical revision of the article. All authors reviewed the manuscript, approved the final draft, and contributed significantly to this work.

\section{FUNDING}

This study was approved by the Guizhou Province Science and Technology Support Project [(2020)4Y165].

\section{ACKNOWLEDGMENTS}

We are particularly grateful to the participators who agreed to participate in our study.

Goularte, J. F., Serafim, S. D., Colombo, R., Hogg, B., Caldieraro, M. A., and Rosa, A. R. (2020). COVID-19 and mental health in Brazil: psychiatric symptoms in the general population. J. Psychiatr. Res. 132, 32-37. doi: 10.1016/j.jpsychires. 2020.09.021

Hammen, C. (2018). Risk factors for depression: an autobiographical review. Annu. Rev. Clin. Psychol. 14, 1-28. doi: 10.1146/annurev-clinpsy-050817-084811

Holden, L., Harris, M., Hockey, R., Ferrari, A., Lee, Y. Y., Dobson, A. J., et al. (2019). Predictors of change in depressive symptoms over time: results from the Australian Longitudinal Study on Women's Health. J. Affect. Disord. 245, 771-778. doi: 10.1016/j.jad.2018.11.076

Hu, H., Cao, Q., Shi, Z., Lin, W., Jiang, H., and Hou, Y. (2018). Social support and depressive symptom disparity between urban and rural older adults in China. J. Affect. Disord. 237, 104-111. doi: 10.1016/j.jad.2018.04.076

Huang, Y., and Zhao, N. (2020). Generalized anxiety disorder, depressive symptoms and sleep quality during COVID-19 outbreak in China: a web-based cross-sectional survey. Psychiatry Res. 288:112954. doi: 10.1016/j.psychres.2020. 112954

Jiang, L., Wang, Y., Zhang, Y., Li, R., Wu, H., Li, C., et al. (2019). The reliability and validity of the center for epidemiologic studies depression scale (CES-D) for Chinese University Students. Front. Psychiatry 10:315. doi: 10.3389/fpsyt.2019. 00315

Karagiannidis, C., Mostert, C., Hentschker, C., Voshaar, T., Malzahn, J., Schillinger, G., et al. (2020). Case characteristics, resource use, and outcomes of 10021 
patients with COVID-19 admitted to 920 German hospitals: an observational study. Lancet Respir. Med. 8, 853-862. doi: 10.1016/s2213-2600(20)30316-7

Keita, M. M., Taverne, B., Sy Savane, S., March, L., Doukoure, M., Sow, M. S., et al. (2017). Depressive symptoms among survivors of Ebola virus disease in Conakry (Guinea): preliminary results of the PostEboGui cohort. BMC Psychiatry 17:127. doi: 10.1186/s12888-017-1280-8

Kozela, M., Bobak, M., Besala, A., Micek, A., Kubinova, R., Malyutina, S., et al. (2016). The association of depressive symptoms with cardiovascular and all-cause mortality in Central and Eastern Europe: prospective results of the HAPIEE study. Eur. J. Prev. Cardiol. 23, 1839-1847. doi: 10.1177/ 2047487316649493

Lai, J., Ma, S., Wang, Y., Cai, Z., Hu, J., Wei, N., et al. (2020). Factors Associated With Mental Health Outcomes Among Health Care Workers Exposed to Coronavirus Disease 2019. JAMA Netw. Open 3:e203976. doi: 10. 1001/jamanetworkopen.2020.3976

Lei, L., Huang, X., Zhang, S., Yang, J., Yang, L., and Xu, M. (2020). Comparison of prevalence and associated factors of anxiety and depression among people affected by versus people unaffected by quarantine during the COVID-19 Epidemic in Southwestern China. Med. Sci. Monit. 26:e924609. doi: 10.12659/ msm.924609

Liu, J., Yan, F., Ma, X., Guo, H. L., Tang, Y. L., Rakofsky, J. J., et al. (2015). Prevalence of major depressive disorder and socio-demographic correlates: results of a representative household epidemiological survey in Beijing, China. J. Affect. Disord. 179, 74-81. doi: 10.1016/j.jad.2015.03.009

Loomba, R. S., Aggarwal, G., Aggarwal, S., Flores, S., Villarreal, E. G., Farias, J. S., et al. (2021). Disparities in case frequency and mortality of coronavirus disease 2019 (COVID-19) among various states in the United States. Ann. Med. 53, 151-159. doi: 10.1080/07853890.2020.1840620

Lopez, E., Steiner, A. J., Manier, K., Shapiro, B. B., Vanle, B., Parisi, T., et al. (2018). Quality of life and functioning of hispanic patients with major depressive disorder before and after treatment. J. Affect. Disord. 225, 117-122. doi: 10.1016/ j.jad.2017.08.031

Mak, I. W., Chu, C. M., Pan, P. C., Yiu, M. G., and Chan, V. L. (2009). Longterm psychiatric morbidities among SARS survivors. Gen. Hosp. Psychiatry 31, 318-326. doi: 10.1016/j.genhosppsych.2009.03.001

Maske, U. E., Buttery, A. K., Beesdo-Baum, K., Riedel-Heller, S., Hapke, U., and Busch, M. A. (2016). Prevalence and correlates of DSM-IV-TR major depressive disorder, self-reported diagnosed depression and current depressive symptoms among adults in Germany. J. Affect. Disord. 190, 167-177. doi: 10.1016/j.jad. 2015.10.006

Meo, S. A., Alhowikan, A. M., Al-Khlaiwi, T., Meo, I. M., Halepoto, D. M., Iqbal, M., et al. (2020). Novel coronavirus 2019-nCoV: prevalence, biological and clinical characteristics comparison with SARS-CoV and MERS-CoV. Eur. Rev. Med. Pharmacol.Sci. 24, 2012-2019. doi: 10.26355/eurrev_202002_20379

Naicker, K., Galambos, N. L., Zeng, Y., Senthilselvan, A., and Colman, I. (2013). Social, demographic, and health outcomes in the 10 years following adolescent depression. J. Adolesc. Health 52, 533-538. doi: 10.1016/j.jadohealth.2012. 12.016

Nelson, B. W., Pettitt, A., Flannery, J. E., and Allen, N. B. (2020). Rapid assessment of psychological and epidemiological correlates of COVID19 concern, financial strain, and health-related behavior change in a large online sample. PLoS One 15:e0241990. doi: 10.1371/journal.pone.024 1990

Onder, G., Rezza, G., and Brusaferro, S. (2020). Case-fatality rate and characteristics of patients dying in relation to COVID-19 in Italy. JAMA 323, 1775-1776. doi: 10.1001/jama.2020.4683

Pasco, J. A., Williams, L. J., Jacka, F. N., Ng, F., Henry, M. J., Nicholson, G. C., et al. (2008). Tobacco smoking as a risk factor for major depressive disorder: population-based study. Br. J. Psychiatry 193, 322-326. doi: 10.1192/bjp.bp.107. 046706

Peng, M., Mo, B., Liu, Y., Xu, M., Song, X., Liu, L., et al. (2020). Prevalence, risk factors and clinical correlates of depression in quarantined population during the COVID-19 outbreak. J. Affect. Disord. 275, 119-124. doi: 10.1016/j.jad.2020. 06.035

Qiu, J., Shen, B., Zhao, M., Wang, Z., Xie, B., and Xu, Y. (2020). A nationwide survey of psychological distress among Chinese people in the COVID19 epidemic: implications and policy recommendations. Gen. Psychiatr. 33:e100213. doi: 10.1136/gpsych-2020-100213
Radloff, L. S. (1977). The CES-D scale: a self-report depression scale for research in the general population. Appl. Psychol. Meas. 1, 385-401. doi: 10.1177/ 014662167700100306

Rodríguez-Rey, R., Garrido-Hernansaiz, H., and Collado, S. (2020). Psychological impact and associated factors during the initial stage of the coronavirus (COVID-19) pandemic among the general population in Spain. Front. Psychol. 11:1540. doi: 10.3389/fpsyg.2020.01540

Rotenstein, L. S., Ramos, M. A., Torre, M., Segal, J. B., Peluso, M. J., Guille, C., et al. (2016). Prevalence of depression, depressive symptoms, and suicidal ideation among medical students: a systematic review and meta-analysis. JAMA 316, 2214-2236. doi: 10.1001/jama.2016.17324

Rousou, E., Kouta, C., and Middleton, N. (2016). Association of social support and socio-demographic characteristics with poor self-rated health and depressive symptomatology among single mothers in Cyprus: a descriptive cross-sectional study. BMC Nurs. 15:15. doi: 10.1186/s12912-016-0134-x

Salari, N., Hosseinian-Far, A., Jalali, R., Vaisi-Raygani, A., Rasoulpoor, S., Mohammadi, M., et al. (2020). Prevalence of stress, anxiety, depression among the general population during the COVID-19 pandemic: a systematic review and meta-analysis. Glob. Health 16:57. doi: 10.1186/s12992-02000589-w

Shi, L., Lu, Z. A., Que, J. Y., Huang, X. L., Liu, L., Ran, M. S., et al. (2020). Prevalence of and risk factors associated with mental health symptoms among the general population in China During the Coronavirus Disease 2019 pandemic. JAMA Netw. Open 3:e2014053. doi: 10.1001/jamanetworkopen.2020.14053

Tadesse, D. B., Gebremeskel, G. G., Asefa, G. G., Abay, M., and Demoz, G. T. (2020). The burden, admission, and outcome of COVID-19 in Africa: protocol for a systematic review and meta-analysis. Emerg. Microbes Infect. 9, 1372-1378. doi: 10.1080/22221751.2020.1775499

Tan, W., Hao, F., McIntyre, R. S., Jiang, L., Jiang, X., Zhang, L., et al. (2020). Is returning to work during the COVID-19 pandemic stressful? A study on immediate mental health status and psychoneuroimmunity prevention measures of Chinese workforce. Brain Behav. Immun. 87, 84-92. doi: 10.1016/j. bbi.2020.04.055

Tang, Y., Serdan, T. D. A., Masi, L. N., Tang, S., Gorjao, R., and Hirabara, S. M. (2020). Epidemiology of COVID-19 in Brazil: using a mathematical model to estimate the outbreak peak and temporal evolution. Emerg. Microbes Infect. 9, 1453-1456. doi: 10.1080/22221751.2020.1785337

The National Health Commission of China (2020). Updates on The Novel Coronavirus Outbreak Up to February 28, 2020. Available: http://www.nhc. gov.cn/xcs/yqtb/202002/4ef8b5221b4d4740bda3145ac37e68ed.shtml (accessed February 29, 2020).

van der Velden, P. G., Contino, C., Das, M., van Loon, P., and Bosmans, M. W. G. (2020). Anxiety and depression symptoms, and lack of emotional support among the general population before and during the COVID-19 pandemic. A prospective national study on prevalence and risk factors. J. Affect. Disord. 277, 540-548. doi: 10.1016/j.jad.2020.08.026

Vilagut, G., Forero, C. G., Barbaglia, G., and Alonso, J. (2016). Screening for depression in the general population with the center for epidemiologic studies depression (CES-D): a systematic review with meta-analysis. PLoS One 11:e0155431. doi: 10.1371/journal.pone.0155431

Wang, C., Horby, P. W., Hayden, F. G., and Gao, G. F. (2020a). A novel coronavirus outbreak of global health concern. Lancet 395, 470-473. doi: 10.1016/s01406736(20)30185-9

Wang, C., Pan, R., Wan, X., Tan, Y., Xu, L., Ho, C. S., et al. (2020b). Immediate psychological responses and associated factors during the initial stage of the 2019 Coronavirus Disease (COVID-19) epidemic among the general population in China. Int. J. Environ. Res. Public Health 17:1729. doi: 10.3390/ ijerph17051729

Whooley, M. A., and Wong, J. M. (2013). Depression and cardiovascular disorders. Annu. Rev. Clin. Psychol. 9, 327-354. doi: 10.1146/annurev-clinpsy-050212185526

World Health Organization (2020a). Statement on The Second Meeting of the International Health Regulations (2005) Emergency Committee Regarding the Outbreak of Novel Coronavirus (2019-nCoV). [Online]. Available online at: https://www.who.int/news/item/30-01-2020-statement-on-thesecond-meeting-of-the-international-health-regulations-(2005)-emergencycommittee-regarding-the-outbreak-of-novel-coronavirus-(2019-ncov) (accessed February 2, 2020). 
World Health Organization (2020b). WHO Director-General's Remarks at the Media Briefing on 2019- $n \mathrm{CoV}$ on 11 February 2020 [Online]. Available: https://www.who.int/dg/speeches/detail/who-director-general-s-remarks-atthe-media-briefing-on-2019-ncov-on-11-february-2020 (accessed February, 11 2020).

Wu, J. T., Leung, K., Bushman, M., Kishore, N., Niehus, R., de Salazar, P. M., et al. (2020). Estimating clinical severity of COVID-19 from the transmission dynamics in Wuhan, China. Nat. Med. 26, 506-510. doi: 10.1038/s41591-020$0822-7$

Wu, Z., and McGoogan, J. M. (2020). Characteristics of and important lessons from the coronavirus disease 2019 (covid-19) outbreak in china: summary of a report of 72314 cases from the chinese center for disease control and prevention. JAMA 323, 1239-1242. doi: 10.1001/jama.2020.2648

Ye, Z. J., Qiu, H. Z., Li, P. F., Chen, P., Liang, M. Z., Liu, M. L., et al. (2017). Validation and application of the Chinese version of the 10-item ConnorDavidson Resilience Scale (CD-RISC-10) among parents of children with cancer diagnosis. Eur. J. Oncol. Nurs. 27, 36-44. doi: 10.1016/j.ejon.2017.01.004

Zhang, J., Yang, Z., Wang, X., Li, J., Dong, L., Wang, F., et al. (2020). The relationship between resilience, anxiety and depression among patients with mild symptoms of COVID-19 in China: a cross-sectional study. J. Clin. Nurs. [Epub ahead of print]. doi: 10.1111/jocn.15425
Zhang, Y. S., Rao, W. W., Cui, L. J., Li, J. F., Li, L., Ng, C. H., et al. (2019). Prevalence of major depressive disorder and its socio-demographic correlates in the general adult population in Hebei province, China. J. Affect. Disord. 252, 92-98. doi: 10.1016/j.jad.2019.01.049

Zhu, N., Zhang, D., Wang, W., Li, X., Yang, B., Song, J., et al. (2020). A novel coronavirus from patients with pneumonia in China, 2019. N. Engl. J. Med. 382, 727-733. doi: 10.1056/NEJMoa2001017

Zimet, G. D., Powell, S. S., Farley, G. K., Werkman, S., and Berkoff, K. A. (1990). Psychometric characteristics of the multidimensional scale of perceived social support. J. Pers. Assess. 55, 610-617. doi: 10.1080/00223891.1990.9674095

Conflict of Interest: The authors declare that the research was conducted in the absence of any commercial or financial relationships that could be construed as a potential conflict of interest.

Copyright $\odot 2020$ Peng, Lai, Du, Li, Tian and Gan. This is an open-access article distributed under the terms of the Creative Commons Attribution License (CC BY). The use, distribution or reproduction in other forums is permitted, provided the original author(s) and the copyright owner(s) are credited and that the original publication in this journal is cited, in accordance with accepted academic practice. No use, distribution or reproduction is permitted which does not comply with these terms. 